\title{
Analysis of chiral amino acids in cerebrospinal fluid samples linked to different stages of Alzheimer disease
}

\author{
Shorena Samakashvili ${ }^{1}$,Clara Ibáñez ${ }^{2}$, Carolina Simó ${ }^{2}$, Francisco J. Gil-Bea ${ }^{3}$, Bengt \\ Winblad $^{3}$, Angel Cedazo-Minguez ${ }^{3}$, Alejandro Cifuentes ${ }^{2, *}$ \\ ${ }^{1}$ Department of Physical and Analytical Chemistry, School of Exact and Natural \\ Sciences, Tbilisi State University, Tbilisi, Georgia \\ ${ }^{2}$ Laboratory of Foodomics, Institute of Food Science Research (CSIC), \\ Nicolas Cabrera 9, 28049 Madrid, Spain. \\ ${ }^{3}$ NVS Department, KI-Alzheimer's Disease Research Center, Karolinska Institute, \\ 14186 Stockholm, Sweden
}

Running title: Chiral MEKC analysis of amino acids from CSF for Alzheimer studies Abreviations: (AD) alzheimer disease, (CD) cyclodextrin, (CNS) central nervous system, (CSF) cerebrospinal fluid, (FITC) fluorescein isothiocyanate, (FSDA) forward stepwise discriminant analysis, (GABA) gamma-aminobutyric acid, (MCI) mild cognitive impairment, (NMDA) $N$-methyl-D-aspartate, (SCI) subjective cognitive impairment,

Keywords: Alzheimer, capillary electrophoresis, cerebrospinal fluid, chiral amino acids.

*Corresponding author: Dr. Alejandro Cifuentes, Fax\#: 34-91-5644853, e-mail: a.cifuentes@csic.es 


\section{ABSTRACT}

Chiral micellar electrokinetic chromatography with laser induced fluorescence detection (chiral-MEKC-LIF) was used to investigate D- and L-amino acids contents in cerebrospinal fluid (CSF) samples related to different Alzheimer disease (AD) stages. CSF samples were taken from i) control subjects (S1 pool), ii) subjects showing a mild cognitive impairment (MCI) who remained stable (S2 pool), iii) subjects showing a MCI that progressed to AD (S3 pool) and iv) subjects diagnosed with AD (S4 pool). The optimized procedure only needed $10 \mu \mathrm{L}$ of CSF and it included sample cleaning, derivatization with FITC and chiral-MEKC-LIF separation. Eighteen standard amino acids were baseline separated with efficiencies up to 703000 plates $/ \mathrm{m}$, high sensitivity (LODs in the nM range) and good resolution (values ranging from 2.6 to 9.5). Using this method, L-Arg, L-Leu, L-Gln, GABA, L-Ser, D-Ser, L-Ala, Gly, L-Lys, L-Glu and L-Asp were detected in all the CSF samples. S3 and S4 samples (i.e., AD subjects) showed significant lower amounts of L-Arg L-Lys, L-Glu and L-Asp compared to the non-Alzheimer S1 and S2 samples, showing the S4 group the lowest amounts of L-Arg L-Lys, L-Glu and L-Asp. Moreover, GABA was significantly higher in AD subjects with the highest amount also found for S4. No significant differences were observed for the rest of amino acids including D-Ser. Based on the obtained chiral-MEKC-LIF data, it was possible to correctly classify all the samples into the four groups. These results demonstrate that the use of enantioselective procedures as the one developed in this work can provide some new light on the investigations of $\mathrm{AD}$, including the discovery of new biomarkers related to different stages of AD.

\section{INTRODUCTION}


Amino acids have focused much attention in biomedical research, medical diagnostics, clinical chemistry, and the pharmaceutical industry, because they play essential roles in control and regulation of crucial functions in the human body [1,2]. Moreover, it has recently been found that L- and D-amino acids can play different biological functions and, therefore, analysis of these chiral forms in biological fluids may provide a means of diagnosis and possible treatment of diseases [3-5]. However, the knowledge of Damino acids in biological samples is still limited and most of the D-amino acids are not well investigated yet, concluding that more work is needed in this area of research [5].

Alzheimer's disease (AD) is a neurodegenerative disorder with an estimated worldwide prevalence of over eighteen million people, and is predicted to increase with an increasing elderly population [6]. AD is characterized by cognitive deficits and memory impairment, and there is currently no cure for this disease. Biochemical processes accounting for neurodegeneration are not known but are likely to include the metabolism of amino acids [6]. Since free amino acids are important in neurotransmission, receptor function and are implicated in neurotoxicity, changes in free amino acids metabolism can be an early indicator of neurodegeneration in AD. This early diagnose is considered crucial for a potential $\mathrm{AD}$ cure because treatment might be most effective when initiated very early in the course of $\mathrm{AD}$, before amyloid plaques and neurodegeneration become too widespread. Thus, biomarkers are needed that can detect $\mathrm{AD}$ in the predementia phase or, ideally, in presymptomatic individuals $[7,8]$. Moreover, in vitro assays have recently shown that aggregation and folding parameters of amyloid beta are stereospecific and the aggregation property strongly depends upon 
the amino acid sequence and their stereospecificity [9], suggesting the stereospecific role of amino acids comprising aggregation and its relevance to neurodegeneration.

Analysis of chiral amino acids can, therefore, be an interesting strategy to further investigate AD. Moreover, analysis of the chiral forms of amino acids can provide more light to the contradictory results usually found in the literature on amino acids analysis related to $\mathrm{AD}$. For instance, some researchers have found no differences in glutamate levels in the hippocampus and cerebral cortices between normal and AD patients [10], whereas other researchers have shown that this amino acid was elevated [11] or reduced $[12,13]$ in brain of AD patients. Similarly, aspartate levels have been found increased [14], reduced $[12,13]$ or virtually unaltered in $\mathrm{AD}$ compared to normal brain [11]. The same differing results are found comparing the published works dealing with the analysis of amino acids in cerebrospinal fluid (CSF) from AD and control subjects using chromatographic [6,15-19] or electrodriven separation techniques [6,20-23]. Though further studies are needed to clarify whether the alterations in amino acids in an AD brain and CSF is a factor or a result, the elucidation of the relationship of amino acids (including their L- and $\mathrm{D}$-forms) and $\mathrm{AD}$ might offer new insights to overcome this devastating disease.

In this regard, chiral capillary electrophoresis (CE) can be a good choice to carry out the analysis of D- and L- amino acids from CSF samples based on CE high separation efficiency, enormous resolving power, large peak capacities, short analysis times and small sample and reagents volume (including tiny amounts of chiral selectors) [24]. Although, as mentioned above, some previous works have already shown the content of amino acids in CSF using different techniques such as HPLC [6,15-17] or CE [6,20,22], 
to our knowledge, only two papers have faced the complex issue regarding the analysis of chiral amino acids in CSF samples related to AD. Namely, Fisher et al. showed in a first work using HPLC that free D-Asp was significantly higher $(p<0.01)$ in AD ventricular CSF compared to normal ventricular CSF [25]. However, in a posterior work, they found that also D-Ser was significantly higher $(\mathrm{p}<0.01)$ in AD ventricular CSF [26]. They concluded that CSF could reflect the degenerative process that occurs in AD since CSF is the repository of amino acids from the brain, although, they state that more work is needed to corroborate this crucial point [26].

The goal of this work is, therefore, to carry out the profiling of the main D- and Lamino acids that can be found in CSF samples related to different stages of AD. To do this, a new analytical CE method is developed combining micellar electrokinetic chromatography (MEKC) with a chiral selector and laser induced fluorescence (LIF) detection to analyze a group of 18 selected L- and D-amino acids that can be found in CSF. The developed chiral-MEKC-LIF method is fast and reproducible and allows the separation of the 18 amino acids with good efficiency and sensitivity.

\section{MATERIALS AND METHODS}

\section{Chemicals}

All chemicals were of analytical reagent grade and used as received. $\beta$-CD was used as chiral selector for the MEKC running buffer together with sodium dodecyl sulfate (SDS) and boric acid, all from Sigma-Aldrich (St. Louis, MO, USA). Water was deionized by using a Milli-Q system from Millipore (Bedford, MA, USA). An aqueous solution containing $5 \mathrm{~mol} / \mathrm{L}$ of sodium hydroxide from Panreac Quimica S.A. 
(Barcelona, Spain) was used to adjust the $\mathrm{pH}$ of the BGEs. A $0.1 \mathrm{~mol} / \mathrm{L} \mathrm{NaOH}$ solution was used to rinse the capillary. BGEs were stored at $4^{\circ} \mathrm{C}$ and warmed at room temperature before use. All solutions were filtrated through a $0.45 \mu \mathrm{m}$ membrane filter. A $20 \mathrm{mM}$ fluorescein isothiocyanate (FITC) solution from Sigma-Aldrich was prepared in acetone from Merck (Darmstadt, Germany). Standard L- and D-amino acids were from Sigma-Aldrich. Stock solutions of $0.05 \mathrm{mM}$ Gly, 0.10 mM D L Arg, D L Ser, D LL

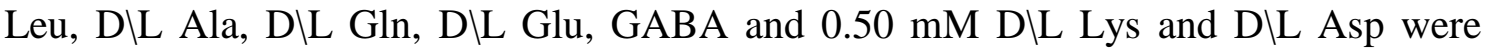
prepared in Milli-Q water. The stock solutions were stored at $4{ }^{\circ} \mathrm{C}$.

\section{CSF samples}

\section{Study population}

The patients included in the study $(n=99)$ were from the Memory Clinic at the Karolinska University Hospital in Huddinge (Sweden). 33 had just subjective cognitive impairment (SCI) and they were considered as control group (sample 1, S1), 39 had mild cognitive impairment (MCI) of which 26 remaining stable (sample 2, S2) and 13 had progression-to-Alzheimer disease within two years time (sample 3, S3), the four group was composed of 27 patients already presenting mild Alzheimer disease (sample

4, S4). These patients were all living independently in the community. They were evaluated according to a standard comprehensive assessment protocol including clinical examination, brain imaging, electroencephalography, analyses of blood and CSF (including total tau (T-Tau), phospho-tau (P-Tau), and $\mathrm{A} \beta_{1-42}$ ) and a detailed neuropsychological evaluation. Dementia and AD were diagnosed according to DSMIV and NINCDS-ADRDA criteria. MCI patients from S2 were not demented, had (self and/or an informant) reported cognitive decline and impairment on objective cognitive 
tasks, and $\mathrm{S} 3$ had preserved basic $\mathrm{AD} /$ minimal impairment in complex instrumental functions. S1 patients had cognitive complaints without impairment on objective cognitive tasks. Patients with psychiatric disorders (i.e. depression, alcohol abuse) or other conditions (i.e. diabetes, brain tumours, normal pressure hydrocephalus) were not included. The study was conducted under the guidelines of the Declaration of Helsinki and approved by the ethics committee of the Karolinska Institutet.

\section{CSF extraction}

CSF samples were obtained by lumbar puncture performed in the sitting position. CSF extraction is routinely performed at the Karolinska University Hospital Memory clinic in Huddinge (Sweden) as part of the medical examination. The extractions were performed in the mornings in fasting patients. CSF samples were obtained from L3/L4 or L4/L5 interspaces after local anaesthetic infiltration in the skin. After disposal of the first $\mathrm{mL}$ the following $10 \mathrm{~mL}$ were collected in polypropylene tubes. No sample contained more than 500 erythrocytes/ $\mu \mathrm{L}$ CSF samples was used. Samples were gently mixed to avoid gradient effects and centrifuged at $2000 \times \mathrm{g}$ for $10 \mathrm{~min}$ to eliminate cells and insoluble material. Supernatants were immediately aliquoted, immediately frozen and stored at $-80^{\circ} \mathrm{C}$ for pending biochemical analyses. In this study, pools of each of the 4 groups were used. Pools were obtained by mixing $10 \mu \mathrm{L}$ of each individual sample, vortex and immediately stored at $-80^{\circ} \mathrm{C}$.

\section{Ultrafiltration}

$100 \mu \mathrm{l}$ of each sample (S1, S2, S3 and S4) was centrifuged at $14000 \times \mathrm{g}$ for $25 \mathrm{~min}$ on a $3 \mathrm{kDa}$ membrane to remove proteins from $\mathrm{CSF}$ samples. The $<3 \mathrm{kDa}$ fraction was used for subsequent FITC derivatization procedure. 


\section{Derivatization procedure}

The FITC derivatization procedure was optimized as described below. The selected conditions consisted of mixing $10 \mu \mathrm{L}$ of the standard solution of amino acids or CSF sample with $80 \mu \mathrm{L}$ of a $25 \mathrm{mM}$ sodium borate buffer at $\mathrm{pH} 10.0$ and $10 \mu \mathrm{L}$ of a $20 \mathrm{mM}$ FITC solution (freshly prepared just before derivatization). The reaction took place overnight in darkness at room temperature. After derivatization, the solution was stored in dark at $4^{\circ} \mathrm{C}$ before chiral-MEKC-LIF analysis.

\section{MEKC-LIF conditions}

All analyses were carried out in triplicate using a P/ACE $2100 \mathrm{CE}$ apparatus from Beckman Instruments (Fullerton, CA, USA) equipped with an Ar+ laser at $488 \mathrm{~nm}$ (excitation wavelength) and $520 \mathrm{~nm}$ (emission wavelength), also from Beckman Instruments. Bare fused-silica capillary was purchased from Composite Metal Services (Worcester, England). The capillary dimensions were $50 \mathrm{~cm}$ of detection length, $57 \mathrm{~cm}$ of total length, and 50- $\mu \mathrm{m}$ i.d. and was thermostated at $30{ }^{\circ} \mathrm{C}$. Injections were made at the anodic end using $\mathrm{N}_{2}$ at $0.5 \mathrm{psi}(3.45 \mathrm{kPa}$ ) for $3 \mathrm{~s}$ (hydrodynamic injection of $3.5 \mathrm{~nL}$ ), and the applied voltage was $+20 \mathrm{kV}$. The P/ACE $2100 \mathrm{CE}$ instrument was controlled by a PC running the System GOLD software from Beckman. Before first use, new capillaries were preconditioned by rinsing with $0.1 \mathrm{M} \mathrm{NaOH}$ for $30 \mathrm{~min}$. The washing protocol between runs was optimized to obtain adequate repeatability, selecting the following conditions: at the beginning of each run, the capillary was rinsed with $0.1 \mathrm{M}$ $\mathrm{NaOH}$ for $2 \mathrm{~min}$, followed by 2 min with Milli-Q water, and then equilibrated for 5 min 
with the running buffer. After optimization, $100 \mathrm{mM}$ sodium tetraborate, $80 \mathrm{mM}$ SDS,

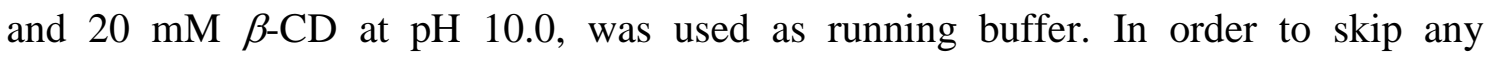
irreproducibility problem derived from buffer depletion, the buffer vial was changed every three injections. At the end of the day, the capillary was rinsed with Milli-Q water for $10 \mathrm{~min}$, and then nitrogen was passed for $2 \mathrm{~min}$.

\section{Data analysis}

Statistical analysis was performed using Statistica 7.1 software (StatSoft Inc.2005, USA, www.statsoft.com). First, one-way analysis of variance (ANOVA) of the amino acid corrected peak areas (i.e., peak area/migration time) was carried out to detect significant differences among the four groups of samples, using a $1 \%$ significance level and a Scheffe test for means comparison. Then a forward stepwise discriminant analysis (FSDA) of the corrected peak areas was applied to select the variables most useful to differentiate the four groups of samples and to obtain the classification functions. Finally, a Fisher's canonical variable analysis (Root 1 and Root 2) was performed in order to obtain a low-dimensional graphical representation of the samples separating as much as possible the groups under study.

\section{RESULTS AND DISCUSSION}

A group of eight chiral amino acids (DL Arg, DL Ser, D L Leu, D L Ala, D L Gln, D L Glu, D L Lys and DLL Asp) plus the nonchiral amino acids Gly and GABA was initially selected to carry out this study. This selection was based on a literature search on the main amino acids usually found in CSF samples. 


\section{Development of the FITC derivatization procedure}

Previous to the optimization of the chiral-MEKC-LIF method, the derivatization procedure was studied in order to achieve the maximum amino acid signal with the lowest number of interferences from the derivatizing reagent FITC, which is known to produce a high number of interfering fluorescent compounds [27]. Besides, different parameters were tested in order to obtain a sensitive and robust derivatizing procedure of the tiny volume of CSF sample used in this work $(10 \mu \mathrm{L})$. Different concentrations of FITC solution were tested (i.e., to give a final concentration equal to 5, 10 and 20 times greater than the concentration of amino acids), different $\mathrm{pHs}$ (from 9 to 11) and concentrations of sodium tetraborate buffer (from $20 \mathrm{mM}$ to $400 \mathrm{mM}$ ) were tested. Optimum results in terms of sensitivity and reproducibility of the derivatization method of the $10 \mu \mathrm{L}$ sample were obtained using a FITC solution with a concentration 10 times greater than the solution of amino acids, and in a $25 \mathrm{mM}$ sodium tetraborate buffer at $\mathrm{pH} 10.0$.

\section{Optimization of chiral-MEKC-LIF separation conditions and figures of merit}

Chiral-MEKC-LIF method was optimized using $\beta$-CD as chiral selector at different concentrations $(10,15$, and $20 \mathrm{mM})$ and testing different SDS concentrations $(40,60$, 80 , and $100 \mathrm{mM}), \mathrm{pH}$ buffer values $(9.5,9.7,10.0,10.3)$ and sodium tetraborate concentrations $(25,50,100,120 \mathrm{mM})$. Optimum chiral resolution was obtained for the 18 amino acids investigated in this work using $100 \mathrm{mM}$ sodium tetraborate, $80 \mathrm{mM}$ SDS and $20 \mathrm{mM} \beta-\mathrm{CD}$ solution at $\mathrm{pH} 10.0$ as BGE. 
A typical electropherogram of the amino acids mixture obtained under these conditions is shown in Figure 1. As can be seen, using this BGE it is possible to achieve the complete chiral-MEKC-LIF separation of D- and L-Arg, D- and L-Leu, D- and L-GIn, GABA, D- and L-Ser, D- and L-Ala, Gly, D- and L-Glu, D- and L-Lys, as well as Dand L-Asp with resolutions higher than 2.6 in all the cases (see Table 1). Thus, these new analytical conditions bring about the baseline separation for the 18 amino acids as clearly shown in Figure 1, besides, they are also well separated from the impurities coming from the FITC derivatization (peaks marked with an asterisk). Moreover, efficiencies ranging from 242000 plates/m for L-Asp to 703000 plates/m for D-Arg were achieved corroborating the usefulness of this procedure. Also, the limits of detection (LOD), calculated considering a signal to noise ratio equal to three, ranged from $0.8 \mathrm{nM}$ for Gly to $16.5 \mathrm{nM}$ for L-Asp, providing limits of quantitation (LOQ) ranging from $2.6 \mathrm{nM}$ for Gly to $53.6 \mathrm{nM}$ for L-Asp. The method was determined to be reproducible according to the results given in Table 2 . Thus, \%RSD values obtained for intra-day repeatability (five consecutive injections in the same day) were better than $5.3 \%$ and $0.5 \%$ for peak areas and migration time, respectively. On the other hand, RSD values obtained for three different days $(n=15)$ were better than $6.6 \%$ and $2.9 \%$ for peak areas and migration time, respectively, assuring an adequate repeatability of the analysis.

In spite of these good results, the present method can sill be improved as can be deduced from the numerous impurities that are obtained from the FITC derivatization (see peaks marked with an asterisk in Figure 1) that can interfere in the determination of other amino acids not included in the standard mixture. However, given the good 
figures of merit in terms of resolution, efficiency and sensitivity of this chiral-MEKCLIF procedure, this method was used to carry out the chiral amino acids profiling in CSF samples.

\section{Identification and relative quantitation of D/L-amino acids in CSF samples related to different AD stages}

Amino acids from CSF samples related to different AD stages were derivatized and analyzed by chiral-MEKC-LIF using the selected conditions described above. The investigated CSF samples were taken from control subjects ( $\mathrm{S} 1$ pool, $\mathrm{n}=33$ individuals), subjects showing a MCI who remained stable (S2 pool, $n=26$ individuals), subjects showing a MCI that progressed to $\mathrm{AD}$ (S3 pool, $\mathrm{n}=13$ individuals) and subjects diagnosed with $\mathrm{AD}$ (S4 pool, $\mathrm{n}=27$ individuals). In order to identify the different enantiomers, a co-injection procedure as shown in Figure 2 was applied allowing the accurate identification of 11 free L/D-amino acids in all the CSF samples. Namely, LArg, L-Leu, L-Gln, GABA, L-Ser, D-Ser, L-Ala, Gly, L-Lys, L-Glu and L-Asp were identified in all the investigated CSF samples. As can be deduced from our results, the only D-amino acid found in CSF was D-Ser. Interestingly, D-Ser is thought to have an important function in the central nervous system (CNS) of mammals to modulate the $\mathrm{N}$ methyl-D-aspartate (NMDA) subtype of glutamate receptor [5,28,29]. Thus, the finding of D-Ser is in good agreement with the fact that only for this D-amino acid it has been possible to demonstrate its natural origin based on the activity of the enzyme serine racemase that catalyzes the direct racemization of L-Ser to D-Ser [5]. So far, there is not

a clear explanation for other D-amino acids that have sometimes been found in 
mammalians including CSF samples, suggesting that they can be derived from nutrition or in many biological samples from bacteria [5].

As already mentioned, further studies are needed to clarify whether the alterations in Damino acids in AD CSF is a factor or a result. In this regard, a correct clinical diagnosis of $\mathrm{AD}$ early in the course of the disease is crucial to initiate symptomatic treatment, and will be even more important when disease-arresting drugs, such as beta-sheet breakers or gamma-secretase inhibitors, will reach the clinic. However, there is no clinical method to determine if a patient with $\mathrm{MCI}$ has incipient $\mathrm{AD}$, i.e. will progress to $\mathrm{AD}$ with dementia, or have a benign form of MCI without progression [30]. Thus, there is a great clinical need for diagnostic biomarkers to identify incipient AD in MCI cases. With this idea in mind, our next step was to inject the CSF samples from the four different groups studied in this work, which included control subjects (S1), subjects showing MCI who remained stable (S2), subjects showing MCI that progressed to AD (S3) and subjects already diagnosed with AD (S4).

The relative levels of the 11 amino acids found in the CSF samples from S1 to S4 groups are given in Table 3, together with their standard deviation after triplicate analysis. Mean values were also compared and the results are included as superscripts. Some interesting differences among the CSF samples are observed in Table 3. Thus, using the statistical approach described in Data analysis (vide supra) the most significant variables $(\mathrm{p}<0.01)$ able to differentiate $\mathrm{S} 1-\mathrm{S} 2$ (non AD subjects) from $\mathrm{S} 3-\mathrm{S} 4$ (AD subjects) were confirmed to be L-Glu, L-Arg, L-Asp, L-Lys and GABA. Thus, S3 and S4 samples (i.e., AD subjects) showed significant $(\mathrm{p}<0.01)$ lower amounts of LArg, L-Lys, L-Glu and L-Asp compared to the non-AD samples S1 and S2, observing in 
the S4 sample the lowest amounts of L-Arg L-Lys and L-Glu. Similar results were mentioned by D'Aniello et al. [17] who observed that aspartic acid occurred at significantly lower concentrations in CSF from AD patients than in CSF from healthy individuals. Also, these results seem to be in good agreement with the excitatory role of some of these amino acids. Thus, as the major excitatory neurotransmitters in the mammalian CNS, Glu and Asp are present in more than half of all CNS synapses, which underscores their important involvement in e.g., learning, memory or movement [31,32]. Based on the observed decrease of these crucial functions in AD patients, it could be expected lower levels of L-Glu and L-Asp in the AD patients as corroborated by results in Table 3 .

Moreover, GABA was significantly higher $(\mathrm{p}<0.01)$ in AD subjects from S3 and $\mathrm{S} 4$ compared to S1 and S2, with the highest amount of GABA also found in S4. Our results do not agree with those reported by Bergquist et al. [20] who analyzed CSF samples from patients with $\mathrm{AD}$ and other neurological diseases observing that GABA levels were reduced in the CSF of $\mathrm{AD}$ patients, while Gly levels in the $\mathrm{AD}$ patients were elevated. This disagreement can be explained considering that GABA and Gly are the main inhibitory neurotransmitters in the CNS [33]. In fact, as many as $10-40 \%$ of nerve terminals in the hippocampus and cerebral cortex may use GABA as a neurotransmitter to transmit "closure" signals [22,34]. Therefore, GABA and/or Gly can be expected to increase in $\mathrm{AD}$ patients considering their main activity as inhibitory neurotransmitters of the CNS. This finding is also supported by Lanctot et al [35] who hypothesized that the variable findings regarding GABA disruption in AD patients would reflect subtypes of this disease that could possibly be manifested clinically by differing behavioural symptoms. 
No significant differences $(\mathrm{p}<0.01)$ were observed for the rest of amino acids including D-Ser. Interestingly, the result on D-Ser does not agree with those reported by Fisher et al. $[25,26]$ who in the 90 's found using HPLC that free D-Ser in CSF from AD patients was significantly higher than in normal subjects. Since then, to our knowledge, the present work is the only published study on the level of D- and L-amino acids in CSF samples related to $\mathrm{AD}$ and it seems to rebut the accepted hypothesis of a higher level of D-Ser in Alzheimer CSF. Moreover, similar results were found by other authors in 1993 [36] and 1995 [37] using HPLC they did not observe significant differences in the level of D-Ser in normal and Alzheimer human brain. In this regard, some authors have mentioned that CSF contamination with erythrocytes could also increase D-Ser concentration [38]. In our opinion, when combining the results from the above studies, there is no consistent D-Ser disruption in AD CSF samples. Thus, D-Ser should not be considered at this moment a reliable diagnostic marker for $\mathrm{AD}$ when analyzing CSF samples.

Although a larger number of samples has to be analyzed in order to confirm the above findings, it is interesting to remark that significant difference in all the investigated samples was only observed for 5 compounds out of the 18 investigated. Thus, other two compounds differing more than statistically expected were Gly and L-Leu. However, the statistically significant difference for Gly and L-Leu is not systematically observed for all the groups as can be deduced from the results given in Table 3.

To confirm the conclusions obtained from the statistical analysis discussed above, all the data as a whole were subjected to a forward stepwise discriminant analysis (FSDA). The data matrix has dimensionality $12 \cdot 7$ (seven chiral-MEKC-LIF peak areas chosen 
from electropherograms of the four CSF groups, S1 to S4, run in triplicate), data analysis was carried out as described in the Experimental Section. In Figure 3, each point represents one particular chiral-MEKC-LIF electropherogram confirming the straightforward statistical analysis performed above. L-Glu, L-Arg, L-Asp, L-Lys and GABA were selected as the most important variables to differentiate the four groups of samples. Values of 4.0 and 3.9 were considered for F-statistic to enter and to remove variables, respectively. With these five amino acids it was possible to classify all samples correctly, as can be seen in Figure 3, the four groups S1, S2, S3 and S4 are nicely separated using this approach. The classification matrix of this analysis reveals L-Glu, L-Asp and L-Lys as crucial for the four groups sorting. The factor structure matrix associated to the FSDA highlight L-Glu and L-Arg as the amino acids which most contributes to $\mathrm{AD}$ and non-AD classification. Namely, S1 and S2 (non AD samples) can be clearly separated from S3 and S4 (AD samples) only by analyzing the levels of these two compounds. Interestingly, the decrease observed in L-Glu and L-Arg in AD CSF samples seems to be in good agreement with the much higher amount of GABA found in the same samples, since GABA is produced from L-Arg via their conversion to ornithine and L-Glu by the action of the enzyme arginase also found in the brain [39-41]. However, some other possible explanations of the decrease of L-Arg in AD CSF samples cannot be ruled out, including a major concentration or activity of enzymes using L-Arg as substrate as e.g., nitric oxide synthase or peptidylarginine deiminases $[41,42]$.

These results seem to demonstrate the good possibilities of chiral-MEKC-LIF analysis of CSF samples as a clinical diagnostic tool of AD even at the early stages of this disease. Moreover, as can be deduced from the good separation between groups S2 and 
S3 it seems that chiral-MEKC-LIF analysis of CSF samples could be proposed as a clinical method to determine if a patient with MCI has incipient AD, i.e. will progress to AD with dementia (group S3), or have a benign form of MCI without progression (group S2). More samples have to be analyzed to corroborate this point.

\section{CONCLUDING REMARKS}

Differences in chiral amino acid levels are clearly observed among the sample pools from different patients examined in this study, indicating that $\mathrm{AD}$ related differences in CSF amino acid profiles can be examined effectively by chiral-MEKC-LIF. Some of the results shown in this work seem to rebut those shown in literature (e.g., not significant variation on D-Ser level was observed depending on the AD stage), while some of them agree with the results obtained by others (e.g., L-Asp occurs at significantly lower concentrations in Alzheimer CSF than normal CSF). Moreover, it is also preliminarily proposed that L-Glu, L-Arg, L-Asp, L-Lys and GABA could be used as biomarkers able to differentiate MCI patients who will progress to AD from those having a benign form of MCI without progression.

From this work, it is concluded that enantioselective procedures can open new perspectives in the discovery of biomarkers related to different stages of AD. However, more CSF samples will have to be analyzed in order to use L-and D-amino acids profiles from chiral-MEKC-LIF to make specific conclusions about neurological disorders such as AD. Moreover, the effect of other conditions (e.g. diet) on the level of D-amino acids found in CSF should also be studied, since the endogenous origin of 
some D-amino acids is already known (i.e., D-Ser), but it is not clear yet the origin of others D-amino acids found in CSF samples in other works.

\section{Acknowledgements}

Sh.S. thanks Shota Rustaveli Georgia National Science Foundation for her Young Scientists Grant (Project \#04/03). C.I. thanks the Ministerio de Ciencia e Innovación for her FPI pre-doctoral fellowship. This work was supported by: Projects AGL200805108-C03-01 and CONSOLIDER INGENIO 2010 CSD2007-00063 FUN-C-FOOD (Ministerio de Educación y Ciencia), Gun och Bertil Stohnes Stiftelse, Karolinska Institutets fund for geriatric research, Stiftelsen Gamla Tjanarinnor, Stiftelsen Dementia, Swedish Alzheimer Foundation, Swedish Brain Foundation, Ramon Areces Foundation and the regional agreement on medical training and clinical research (ALF) between Stockholm County Council and the Karolinska Institute. 


\section{REFERENCES}

[1] Wu, G.Y., Amino acids. 2009, 37, 1-17.

[2] Arthur, R., J. Comp. Med. 2009, 8, 46-61.

[3] Kirschner, D. L., Green, T. K., J. Sep. Sci. 2009, 32, $2305-2318$.

[4] Hamase, K., Morikawa, A., Etoh, S., Anal. Sci. 2009, 25, 961-968.

[5] Hamase, K., Morikawa, A., Zaitsu, K., J. Chromatogr B. 2002, 781, 73-91.

[6] Fonteh, A. N., Harrington, R. J., Tsai, A., Liao, P., Harrington, M. G., Amino Acids 2007, 32, 213-224.

[7] Blennow, K., Hampel, H., Weiner, M., Zetterberg, H., Nature Rev. Neurol. 2010, $6,131-144$.

[8] Cedazo-Minguez, A., Winblad, B., Exp Gerontol. 2010, 45, 5-14.

[9] Gupta, V.B., Indi, S. S., Rao, K. S. J., J. Mol. Neurosci. 2008, 34, 35-43.

[10] Tarbit, I., Perry, E. K., Perry, R. H., Blessed, G., Tomlinson, B. E., J. Neurochem. 1980, 35, 1246-1249.

[11] Procter, A. W., Lowe, S. L., Palmer, A. M., Francis, P. T., Esiri, M. M., Stratmann, G. C., Najlerahim, A., Patel, A. J., Hunt, A., Bowen, D. M., J. Neurol. Sci. 1988, 84, 125-140.

[12] Elison, D. W., Beal, M. F., Mazurek, M. F., Bird, E. D., Martin, J. B., Ann. Neurol. 1986, 20, 616-621.

[13] Sasaki, H., Muromoto, O., Komzowa, I., Arai, H., Kosaka, K., Iizuka, R., Ann. Neurol. 1986, 19, 263-269.

[14] Perry, E. K., Atack, J. R., Perry, R. H., Hardy, J. A., Dodd, P. R., Edwardson, J. A., Blessed, G., Tomlinson, B. E., Fairbairn, A. F., J. Neurochem. 1984, 42, 14021410.

[15] Kaiser, E., Schoenknecht, P., Kassner, S., Hildebrandt, W., Kinscherf, R., Schroeder., J. Neurodegen. Diseases. 2010, 7, 251-259.

[16] Molina, J. A., Jiménez-Jiménez, F. J., Vargas, C., Gómez, P., de Bustos, F., Ortí-Pareja, M., Tallón-Barranco, A., Benito-León, J., Arenas, J., Enríquez-deSalamanca, R., J. Neural Transm. 1998, 105, 279-286.

[17] D’Aniello, A., Fisher, G., Migliaccio, N., Cammisa, G., D’Aniello, E., Spinelli, P., Neurosci. Lett. 2005, 388, 49-53.

[18] Mochizuki, Y., Oishi, M., Hara, M., Takasu, T., Annals Clin. Lab. Sci. 1996, $26,275-278$. 
[19] Martinez, M., Frank, A., Dieztejedor, E., Hernanz, A., J. Neural Transm. 1993, $6,1-9$.

[20] Bergquist, J., Gilman, D., Ewing, A. G., Ekman, R., Anal. Chem. 1994, 66, 3512-3518.

[21] Thorsen, G., Bergquist, J., J. Chromatgr. B. 2000, 745, 389-397.

[22] Deng, Y. H., Wang, H., Zhang, H. S., J. Sep. Sci. 2008, 31, 3088-3097.

[23] Lu, M. J., Chiu, T. C., Chang, P. L., Hoc, H. T., Chang, H. T., Anal. Chim. Acta. 2005, 538, 143-150.

[24] Herrero, M., Simó, C., García-Cañas, V., Fanali, S., Cifuentes, A., Electrophoresis. 2010, 31, 2106-2114.

[25] Fisher, G. H., Petrucelli, L., Gardner, C., Emory, C., Frey, W. H., Amaducci, L., Sorbi, S., Sorrentino, G., Borghi, M., D’Aniello, A., Mol. Chem. Neuropathol. 1994, 23, 115-124.

[26] Fisher, G., Lorenzo, N., Abe, H., Fujita, E., Frey, W. H., Emory, C., Fiore, M. M. D., D’Aniello, A., Amino Acids. 1998, 15, 263-269.

[27] Simó, C., Barbas, C., Cifuentes, A., J. Agric. Food Chem. 2002, 50, 5288-5293. [28] Mothet, J. P., Pathol. Biol. 2001, 49, 655-659.

[29] Barañano, D. E., Ferris, C. D., Snyder, S. H., Trends Neurosci. 2001, 24, 99106.

[30] Blennow, K., J. Int. Med. 2004, 256, 224-234.

[31] Rawls, S. M., Gomez, T., Stagliano, G. W., Raffa, R. B., J. Pharmacol. Toxicol. Methods. 2006, 53, 291-295.

[32] Boyd, B. W., Witowski, S. R., Kennedy, R. T., Anal. Chem. 2000, 72, 865-871.

[33] Piepponen, T. P., Skujins, A., J. Chromatogr. B 2001, 757, 277-283.

[34] Shaha, A. J., Crespib, F., Heidbrederb, C., J. Chromatogr. B. 2002, 781, 151163.

[35] Lanctot, K. L., Herrmann, N., Mazzotta, P., Khan, L. R., Ingber, N., Canadian J. Psych. 2004, 49, 439-453.

[36] Chouinard, M. L., Gaitan, D., Wood, P. L., J. Neurochem. 1993, 61, 15611564.

[37] Nagata, Y., Borghi, M., Fisher, G. H., D’Aniello, A., Brain Res.Bull. 1995, 38, 181-183. 
[38] Fuchs, S.A., de Sain-van der Velden, M. G. M., de Barse, M. M. J., Roeleveld M. W., Hendriks, M., Dorland, L., Klomp, L. W. J., Berger, R., de Koning, T., J. Clinical Chem. 2008, 54, 1443-1450.

[39] Albina, J. E., Mills, C. D., Henry, W. L., Caldwell, M. D., J. Immunol. 1990, 144, 3877-3880.

[40] Jenkinson, C. P., Grody, W. W., Cederbaum, S. D., Comp Biochem Physiol B Biochem Mol Biol. 1996, 114, 107-132.

[41] Vural, H., Sirin, B., Yilmaz, N., Eren, I., Delibas, N., Biol. Trace Elem. Res. 2009, 129, 58-64.

[42] Ishigami, A., Maruyama, N., Geriatr. Gerontol. Int. 2010, 10, 53-58. 


\section{FIGURE LEGENDS}

Figure 1. MEKC-LIF electropherograms of a standard mixture of $18 \mathrm{D} / \mathrm{L}$ amino acids $(10 \mu \mathrm{g} / \mathrm{mL}$ D/L-Arg, D/L-Leu, D/L-Gln, GABA, D/L-Ser, D/L-Ala, D/L-Glu, $5 \mu \mathrm{g} / \mathrm{mL}$ Gly, $50 \mu \mathrm{g} / \mathrm{mL}$ D/L-Lys and D/L-Asp). Peaks marked with an asterisk correspond to FITC peaks. Background electrolyte: $100 \mathrm{mM}$ sodium tetraborate, 80 $\mathrm{mM}$ SDS, $20 \mathrm{mM} \beta$-CD at $\mathrm{pH}$ 10.0. FITC-derivatized amino acids injected for $3 \mathrm{~s}$ at $0.5 \mathrm{psi}$; bare fused-silica capillary with $57 \mathrm{~cm}$ total length, $50 \mathrm{~cm}$ detection length, and $50 \mu \mathrm{m}$ i.d.; running voltage, $20 \mathrm{kV}$; LIF detection, Ar+ laser at $488 \mathrm{~nm}$ (excitation wavelength) and $520 \mathrm{~nm}$ (emission wavelength).

Figure 2. MEKC-LIF analysis of CSF sample S2 (solid trace A) and CSF sample spiked with D/L-Leu, D/L-Gln, GABA, D/L-Ser, D/L-Ala and Gly (dotted trace B). Peaks marked with * correspond to FITC-related peaks and those marked with ** correspond to unidentified peaks from CSF sample. All the analytical conditions as in Figure 1.

Figure 3. Scatter plot of the chiral-MEKC-LIF electropherograms (each point represents one electropherogram) in the plane defined by the two canonical variables (Root 1 and Root 2) obtained from FSDA selected variables: L-Glu, LArg, L-Asp, L-Lys and GABA. These amino acids classify successfully (100\% of classification for all groups) the four pooled samples: S1 (control CSF), S2 (stable $\mathrm{MCI}$ ), S3 (MCI with progression to AD) and S4 (AD). Ellipses surrounding the four groups are drawn for a better visualization. See text for more details. 
Table 1. Figures of merit of the chiral-MEKC-LIF method developed in this work.

\begin{tabular}{|c|c|c|c|}
\hline Amino acid & LOD $(\mathbf{n M})^{\mathbf{a})}$ & Efficiency $^{\text {b) }}$ & Resolution $^{c)}$ \\
\hline D-Arg & 1.6 & 703000 & \multirow{2}{*}{2.6} \\
\hline L-Arg & 1.6 & 686000 & \\
\hline D-Leu & 0.9 & 492000 & \multirow{2}{*}{7.0} \\
\hline L-Leu & 0.7 & 467000 & \\
\hline D-Gln & 0.9 & 433000 & \multirow{2}{*}{4.4} \\
\hline L-Gln & 1.0 & 440000 & \\
\hline GABA & 1.1 & 419000 & - \\
\hline D-Ser & 1.9 & 441000 & \multirow{2}{*}{3.5} \\
\hline L-Ser & 1.9 & 444000 & \\
\hline D-Ala & 2.0 & 363000 & \multirow{2}{*}{4.0} \\
\hline L-Ala & 2.3 & 417000 & \\
\hline Gly & 0.8 & 384000 & - \\
\hline D-Glu & 3.4 & 299000 & \multirow{2}{*}{9.5} \\
\hline L-Glu & 4.4 & 282000 & \\
\hline D-Lys & 11.7 & 292000 & \multirow{2}{*}{2.7} \\
\hline L-Lys & 12.3 & 297000 & \\
\hline D-Asp & 15.4 & 272000 & \multirow{2}{*}{7.3} \\
\hline L-Asp & 16.5 & 242000 & \\
\hline
\end{tabular}

a) Limit of detection (LOD) calculated for a signal/noise ratio of 3 .

b) Efficiency $(\mathrm{N})$ calculated as number of theoretical plates per meter of column.

c) Resolution (R) between two peaks (namely, 1 and 2) was calculated using the next formula: $\mathrm{R}=\left(\mathrm{t}_{2}-\mathrm{t}_{1}\right) /\left(\mathrm{w}_{2}-\mathrm{w}_{1}\right)$, where $\mathrm{t}_{1}$ and $\mathrm{t}_{2}$ are the analysis time of enantiomers 1 and 2 , respectively, and $\mathrm{w}_{1}$ and $\mathrm{w}_{2}$ are the widths at the base of enantiomers 1 and 2, respectively. 
Table 2. Time analysis and corrected peak area reproducibility (determined as relative standard deviation, \%RSD) of the chiral-MEKC-LIF method for the same day $(n=5)$ and three different days $(n=15)$.

\begin{tabular}{|c|c|c|c|c|}
\hline \multirow{2}{*}{ amino acid } & \multicolumn{2}{|c|}{$\%$ RSD (intraday) } & \multicolumn{2}{c|}{$\%$ RSD (three days) } \\
\cline { 2 - 5 } & $\mathrm{t}_{\text {mig }}$ & $\begin{array}{c}\text { Corrected } \\
\text { peak area }\end{array}$ & $\mathrm{t}_{\mathrm{mig}}$ & $\begin{array}{c}\text { Corrected } \\
\text { peak area }\end{array}$ \\
\hline D-Arg & 0.3 & 3.8 & 2.0 & 5.0 \\
\hline L-Arg & 0.3 & 4.2 & 2.0 & 5.0 \\
\hline D-Gln & 0.3 & 3.0 & 2.1 & 5.2 \\
\hline L-Gln & 0.5 & 3.9 & 2.1 & 5.8 \\
\hline D-Ser & 0.5 & 2.8 & 2.3 & 5.9 \\
\hline L-Ser & 0.4 & 4.5 & 2.4 & 6.1 \\
\hline D-Asp & 0.5 & 4.8 & 2.7 & 6.3 \\
\hline L-Asp & 0.3 & 5.3 & 2.9 & 6.6 \\
\hline
\end{tabular}


Table 3. Mean values ( \pm standard deviation) of relative levels of L-and D-amino acids found in the four different CSF pools analyzed. CSF samples were taken from control subjects (S1 pool), subjects showing a mild cognitive impairment (MCI) who remained stable (S2 pool), subjects showing a MCI that progressed to AD (S3 pool) and subjects diagnosed with AD (S4 pool).

\begin{tabular}{|c|c|c|c|c|}
\hline Amino & CSF-S1 pool & CSF-S2 pool & CSF-S3 pool & CSF-S4 pool \\
\hline acid & Rel. level $^{1)}( \pm \mathrm{SD})^{2)}$ & Rel. level $( \pm$ SD) & Rel. level $( \pm$ SD) & Rel. level ( \pm SD) \\
\hline D-Arg & $\mathrm{ND}^{3)}$ & ND & ND & ND \\
\hline L-Arg & $1423^{b} \pm 113$ & $1243^{b} \pm 63$ & $868^{a} \pm 44$ & $802^{a} \pm 16$ \\
\hline D-Leu & ND & ND & ND & ND \\
\hline L-Leu & $839^{\mathrm{a}} \pm 51$ & $1107^{b} \pm 108$ & $1253^{b} \pm 40$ & $1295^{\mathrm{b}} \pm 40$ \\
\hline D-Gln & ND & ND & ND & ND \\
\hline L-Gln & $9174^{\mathrm{a}} \pm 449$ & $9373^{a} \pm 101$ & $8933^{a} \pm 166$ & $9161^{\mathrm{a}} \pm 120$ \\
\hline GABA & $1137^{\mathrm{a}} \pm 108$ & $1370^{\mathrm{ab}} \pm 22$ & $1611^{b c} \pm 22$ & $1805^{\mathrm{c}} \pm 162$ \\
\hline D-Ser & $274^{\mathrm{a}} \pm 17$ & $266^{\mathrm{a}} \pm 23$ & $236^{\mathrm{a}} \pm 21$ & $239^{\mathrm{a}} \pm 13$ \\
\hline L-Ser & $779^{a} \pm 30$ & $769^{a} \pm 22$ & $731^{a} \pm 36$ & $715^{a} \pm 25$ \\
\hline D-Ala & ND & ND & ND & ND \\
\hline L-Ala & $1295^{\mathrm{a}} \pm 134$ & $1302^{\mathrm{a}} \pm 57$ & $1258^{\mathrm{a}} \pm 114$ & $1189^{\mathrm{a}} \pm 47$ \\
\hline Gly & $588^{b} \pm 51$ & $328^{a} \pm 12$ & $332^{\mathrm{a}} \pm 15$ & $298^{a} \pm 29$ \\
\hline D-Lys & ND & ND & ND & ND \\
\hline L-Lys & $173^{c} \pm 5$ & $160^{\mathrm{bc}} \pm 11$ & $139^{b} \pm 10$ & $104^{\mathrm{a}} \pm 7$ \\
\hline D-Glu & ND & ND & ND & ND \\
\hline L-Glu & $87^{b} \pm 4$ & $84^{b} \pm 2$ & $43^{\mathrm{a}} \pm 6$ & $39^{\mathrm{a}} \pm 2$ \\
\hline D-Asp & ND & ND & ND & ND \\
\hline L-Asp & $29^{b} \pm 2$ & $30^{b} \pm 2$ & $17^{\mathrm{a}} \pm 1$ & $19^{\mathrm{a}} \pm 1$ \\
\hline
\end{tabular}

${ }^{1)}$ Relative level given in arbitrary units and calculated as corrected peak area (peak area/migration time).

2) Standard deviation $(\mathrm{n}=3)$.

${ }^{3)}$ Not detected.

${ }^{\mathrm{a}-\mathrm{d}}$ : rows without a common letter are significantly different $(\mathrm{p}<0.01)$ 


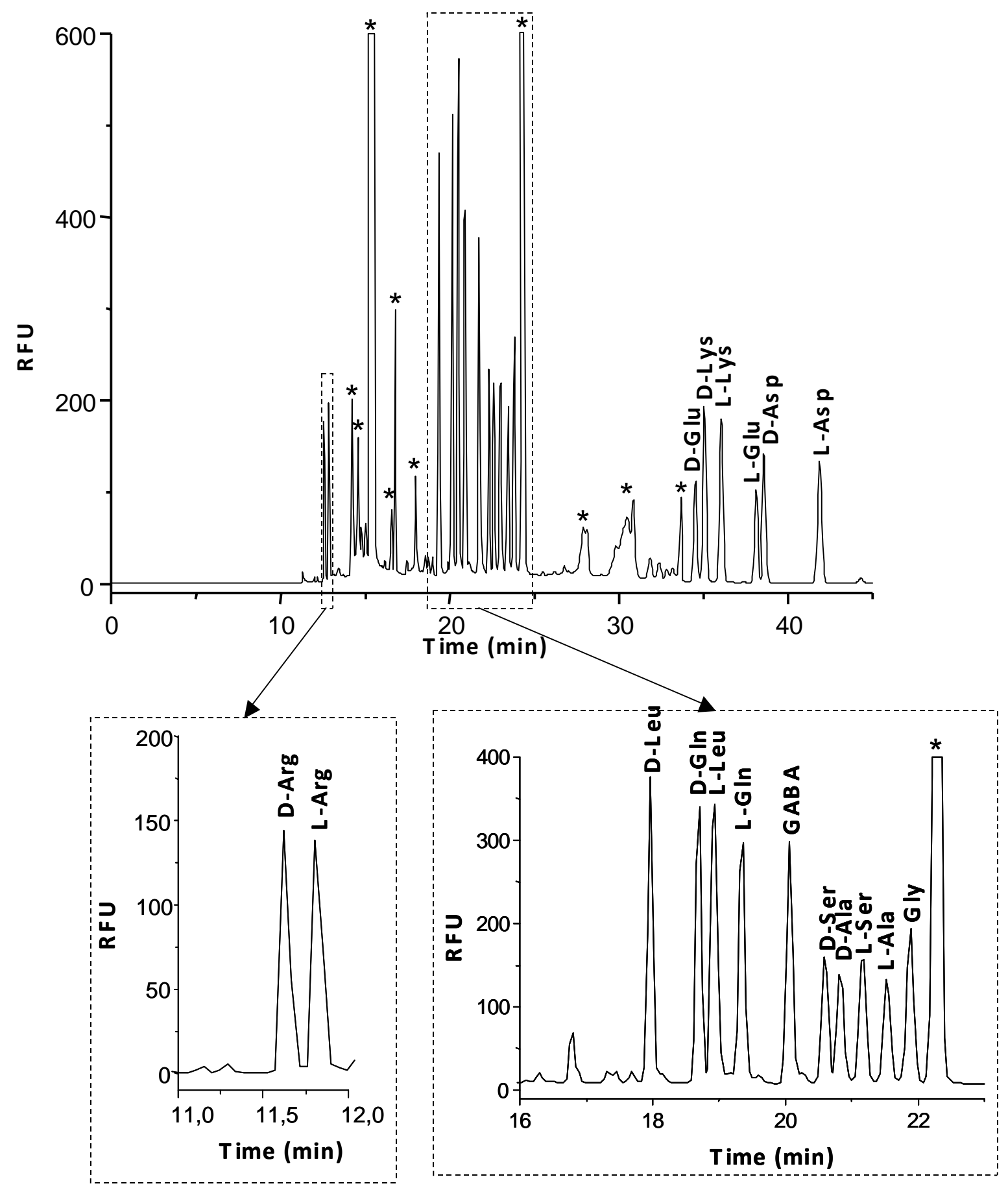

Figure 1 


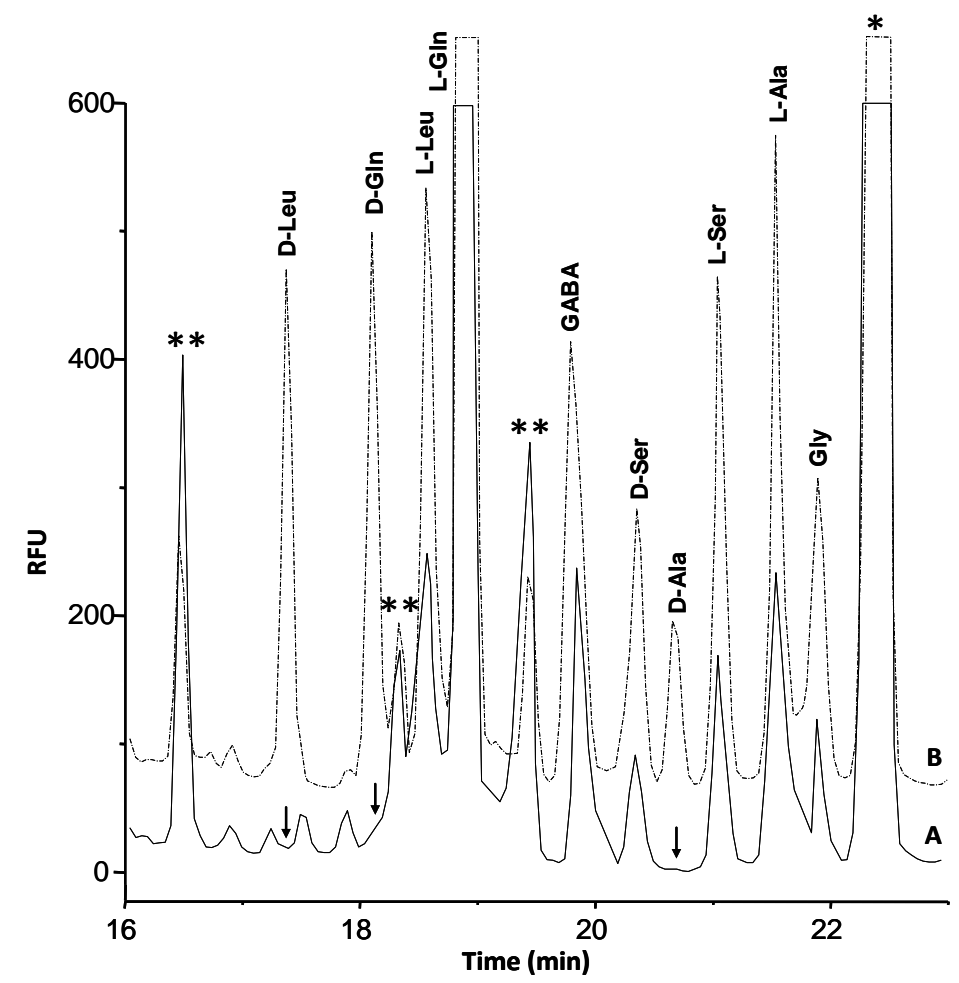

Figure 2 
Root 1 vs. Root 2

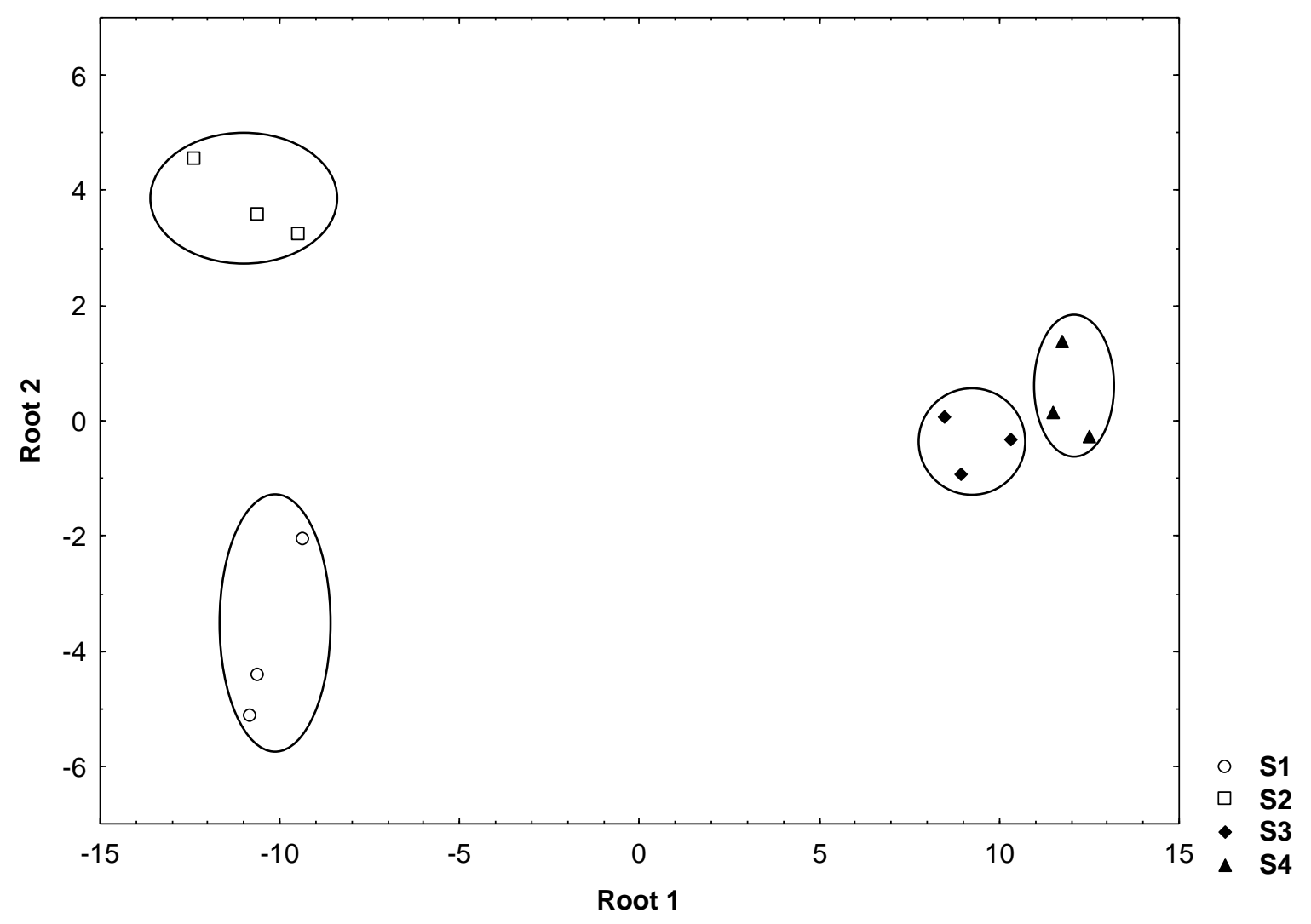

Figure 3 